\title{
Kinetic model for mechanical twinning and its application for intensive loading of metals
}

\author{
Alexander Mayer ${ }^{1, a}$ and Elijah Borodin ${ }^{2,1}$ \\ ${ }^{1}$ Chelyabinsk State University, Department of General and Applied Physics, 454001 Br. Kashirinykh str. 129, \\ Chelyabinsk, Russia \\ 2 IPME RAS, Extreme States Dynamics Department, 199178 Bolshoj pr. V.O., 61 St. Petersburg, Russia
}

\begin{abstract}
In this report, we present our twinning model intended for simulation of the dynamic deformation of metals with low values of the stacking fault energy, as well as the results of application of the model to numerical simulation of intensive loading of metals. Generation of a twin is described as an appearance of a stacking fault with size more than some critical value, while growth of a twin is considered as a cooperative movement of partial dislocation along the stacking fault. The twin nucleation rate is expressed through the energy released due to annihilation of dislocations. Movement of partial dislocations in the course of twin growth passes under the action of elastic stress field and phonon drag. The surface energy of the growing twin continuously increases which leads to the appearance of an additional force. Application of this model allows us to investigate plastic response of metals at various dynamic loading conditions and initial defect structures. Influence of twinning at Taylor rod compaction experiments is analyzed including formation of the shape of the lateral surface.
\end{abstract}

\section{Introduction}

Dislocation plasticity [1-5] is the main mechanism of the plastic deformation of metals in most cases. Twinning becomes an alternative plasticity mechanism at low temperatures and high strain rates $[1,6,7]$; it occurs if value of the stacking fault energy is less than $100 \mathrm{~mJ} / \mathrm{m}^{2}$, which is the case for copper, steel and for many alloys. Mechanical twinning reveals itself in many dynamical processes including the Taylor impact tests $[8,9]$ applied to a metal with a low value of the stacking fault energy.

There are a lot of dislocation plasticity models including applicable for the dynamic conditions of deformation [3-5]. At the same time, the majority of the existing twinning models are designed for quasistatic conditions; most of the models are empirical and parameterized by a number of adjustable parameters.

In this report, we present our twinning model intended for simulation of the dynamic deformation of metals with low values of the stacking fault energy, as well as the results of application of the model to numerical simulation of intensive loading of metals. The model is based on the kinetics of twinning and takes into account generation of new and growth of existing twins.

\section{Mathematical model}

Total plastic strain is represented as a result of the combined action of two competing processes: the dislocation motion and the mechanical twinning. The plastic deformation tensor $w_{i k}$ is represented by a sum: $w_{i k}=$ $w_{i k}^{\mathrm{D}}+w_{i k}^{\mathrm{T}}$, where $w_{i k}^{\mathrm{D}}$ is caused by the dislocation motion and $w_{i k}^{\mathrm{T}}$ is caused by the mechanical twinning. These

\footnotetext{
${ }^{a}$ Corresponding author: mayer@csu.ru
}

tensors are determined through evolution of corresponding defect subsystems. For dislocation plasticity $w_{i k}^{\mathrm{D}}$ we use the model proposed in $[4,5]$.

\subsection{Continuum mechanics equations}

The common part of the mathematical model consists of three conservation laws. The first one of them is the continuity equation:

$$
\frac{1}{\rho} \frac{d \rho}{d t}=-\sum_{k=1}^{N} \frac{\partial v_{k}}{\partial x_{k}},
$$

where $\rho$ is the substance density; $v_{k}$ is the velocity vector; $x_{k}$ is the Cartesian coordinates; $N$ is the number of dimensions of the considered problem, index $k$ numerates spatial directions. The total time derivatives are used, which is valid for Lagrangian particles moving with substance. The equation of substance motion:

$$
\rho \frac{d v_{i}}{d t}=-\frac{\partial P}{\partial x_{i}}+\sum_{k=1}^{N} \frac{\partial S_{i k}}{\partial x_{k}}, \quad i=1, \ldots, N,
$$

where $P$ is the pressure, which is determined from a wide-range equation of state $P=P(\rho, U)[10] ; S_{i k}$ is the tensor of stress deviators, which characterizes the shear stresses; $U$ is the internal energy determined from the energy conservation law in the following form:

$$
\rho \frac{d U}{d t}=\frac{P}{\rho} \frac{d \rho}{d t}+\sum_{i=1}^{N} \sum_{k=1}^{N} S_{i k} \frac{d w_{i k}}{d t},
$$

where the second term in the right-hand part is the heat release due to the plastic deformation. 
The generalized Hooke law [11] with accounting of the plastic strain $w_{i k}$ is used for determination of the stress deviators:

$$
S_{i k}=2 G\left[u_{i k}-\frac{1}{3} \delta_{i k} \sum_{l=1}^{N} u_{l l}-w_{i k}\right], \quad i, k=1, \ldots, N,
$$

where $G$ is the shear modulus, $\delta_{i k}$ is the bivalent mixed tensor, $u_{i k}$ is the tensor of macroscopic deformations:

$$
\begin{aligned}
& \frac{d u_{i k}}{d t}=\frac{1}{2}\left[\frac{\partial v_{i}}{\partial x_{k}}+\frac{\partial v_{k}}{\partial x_{i}}\right]+ \\
& +\frac{1}{2} \sum_{l=1}^{N}\left\{u_{i l}\left(\frac{\partial v_{k}}{\partial x_{l}}-\frac{\partial v_{l}}{\partial x_{k}}\right)+u_{l k}\left(\frac{\partial v_{i}}{\partial x_{l}}-\frac{\partial v_{l}}{\partial x_{i}}\right)\right\}
\end{aligned}
$$

where the first term in the right-hand part is the infinitesimal strain rate tensor; the second term accounts for the change of the tensor components in the laboratory coordinate system due to the substance rotation.

\subsection{Model of twinning}

Plastic deformation caused by twinning can be expressed through the volume fraction of twins:

$$
\begin{gathered}
\frac{d w_{i k}^{\mathrm{T}}}{d t}=\sum_{\gamma} \frac{d \alpha^{\gamma}}{d t}\left(\tau_{i}^{\gamma} n_{k}^{\gamma}+\tau_{k}^{\gamma} n_{i}^{\gamma}\right) \varepsilon_{\mathrm{T}}+ \\
+\frac{1}{2} \sum_{l=1}^{N}\left\{w_{i l}^{\mathrm{T}}\left(\frac{\partial v_{k}}{\partial x_{l}}-\frac{\partial v_{l}}{\partial x_{k}}\right)+w_{l k}^{\mathrm{T}}\left(\frac{\partial v_{i}}{\partial x_{l}}-\frac{\partial v_{l}}{\partial x_{i}}\right)\right\}(6)
\end{gathered}
$$

where $\alpha^{\gamma}$ is the volume fraction of twinned material; $\varepsilon_{\mathrm{T}}$ is the deformation of twinned material with respect to the initial one, for example $\varepsilon_{\mathrm{T}}=1 / \sqrt{2}$ in FCC metals [6]; unit vectors $\tau_{k}^{\gamma}$ and $n_{k}^{\gamma}$ describe the crystallographic orientations of twins; index $\gamma$ numerates all possible orientations. The second term in the right-hand part plays the same role as in Eq. (5). We suppose that twins are cylindrical and have the same radius and thickness in a physically small substance element, than the volume fraction is equal to

$$
\alpha^{\gamma}=N_{\mathrm{T}}^{\gamma} \cdot\left(\pi\left(R_{\mathrm{T}}^{\gamma}\right)^{2} \cdot h_{\mathrm{T}}^{\gamma}\right)+N_{\mathrm{I}}^{\gamma} \cdot\left(\pi\left(R_{\mathrm{I}}^{\gamma}\right)^{2} \cdot h_{\mathrm{I}}^{\gamma}\right),
$$

where $N_{\mathrm{T}}^{\gamma}$ and $N_{\mathrm{I}}^{\gamma}$ are the local concentrations of twins, mobile and immobilized correspondently; $R_{\mathrm{T}}^{\gamma}$ and $R_{\mathrm{I}}^{\gamma}$ are radiuses of mobile and immobilized twins; $h_{\mathrm{T}}^{\gamma}$ and $h_{\mathrm{I}}^{\gamma}$ are their thicknesses. All these values are determined by the kinetics of twins.

Energy of a twin consists of the surface part determined by the stacking fault energy $\gamma_{\mathrm{T}}$, the energy in external stresses and the energy of elastic deformations of the surrounding matrix [12]. Differentiation of this energy over radius or thickness gives the forces tending to change the corresponding size of the twin:

$$
\begin{aligned}
F_{R}^{\gamma}= & 4 \pi \varepsilon_{\mathrm{T}} \cdot R_{\mathrm{T}}^{\gamma} h_{\mathrm{T}}^{\gamma} \cdot \sum_{k=1}^{N} \sum_{i=1}^{N} S_{i k} n_{i}^{\gamma} \tau_{k}^{\gamma}- \\
& -2 \pi \gamma_{\mathrm{T}} \cdot\left(2 R_{\mathrm{T}}^{\gamma}+h_{\mathrm{T}}^{\gamma}\right)-\Phi \cdot\left(h_{\mathrm{T}}^{\gamma}\right)^{2},
\end{aligned}
$$

$$
\begin{aligned}
F_{h}^{\gamma}= & 2 \pi \varepsilon_{\mathrm{T}} \cdot\left(R_{\mathrm{T}}^{\gamma}\right)^{2} \cdot \sum_{k=1}^{N} \sum_{i=1}^{N} S_{i k} n_{i}^{\gamma} \tau_{k}^{\gamma}- \\
& -2 \pi \gamma_{\mathrm{T}} \cdot R_{\mathrm{T}}^{\gamma}-2 \Phi \cdot R_{\mathrm{T}}^{\gamma} h_{\mathrm{T}}^{\gamma},
\end{aligned}
$$

where $\Phi=2 \pi^{3}(2-v) /[3(1-v)] G \varepsilon_{T}^{2}$ and $v$ is the Poisson ratio. Growth of the twin radius or thickness is connected with motion of twinning dislocations at its edges of the twin. The balance between the forces (8), (9) and the drag forces acting on these dislocations allows one to find the growth equations:

$$
\frac{d R_{\mathrm{T}}^{\gamma}}{d t}=F_{R}^{\gamma} / B, \quad \frac{d h_{\mathrm{T}}^{\gamma}}{d t}=F_{h}^{\gamma} / B
$$

where $B$ is the drag coefficient.

Expressions (8) and (9) give the critical radius $R_{\mathrm{c}}^{\gamma}$ and thickness $h_{\mathrm{c}}^{\gamma}$ of twin at which forces are equal to zero-a twin larger than the critical one will grow up. Immobilized twins can not grow in radius but they can become thicker similar to Eqs. (9), (10).

Kinetics equations are written from the energy-wise consideration. The twinning becomes an active channel of plastic deformation when the dislocation plasticity becomes ineffective. Effectiveness of the dislocation channel is restricted by annihilation of dislocations. A certain part of the plastically dissipated energy is spent on the formation of new defects $[13,14]$. In the conditions of active annihilation, the dissipated energy can not be stored in the dislocation subsystem and should be transmitted to anther type of defects, twins for example. Thus, the generation rate of twins is as follows:

$$
\dot{N}_{\mathrm{TW}}^{\gamma+}=\varepsilon_{\mathrm{D}} Q_{\mathrm{a}}^{\beta} /\left(4 \pi\left(R_{\mathrm{c}}^{\gamma}\right)^{2} \gamma_{\mathrm{T}} \cdot \gamma_{0}\right),
$$

where $Q_{a}$ is the total annihilation rate of dislocations; $\gamma_{0}$ is the total number of possible orientations of twins. A twin becomes immobilized then it reaches a grain boundary or another twin; the immobilization rate can be written as follows:

$$
\dot{N}_{\mathrm{T}}^{\gamma-}=N_{\mathrm{T}}^{\gamma} \cdot\left|d R_{\mathrm{T}}^{\gamma} / d t\right| \cdot\left(\Delta^{-1}+d^{-1}\right),
$$

where $d$ is the grain diameter. The average distance between the twins $\Delta$ is connected with their volume fraction [6] $\Delta^{-1}=\sum_{\gamma}\left[\alpha^{\gamma} /\left\{\left(1-\alpha^{\gamma}\right) h_{\mathrm{T}}^{\gamma}\right\}\right]$. Finally, the kinetics equations are the following

$$
\frac{d N_{\mathrm{T}}^{\gamma}}{d t}=\dot{N}_{\mathrm{T}}^{\gamma+}-\dot{N}_{\mathrm{T}}^{\gamma-} \frac{d N_{\mathrm{I}}^{\gamma}}{d t}=\dot{N}_{\mathrm{T}}^{\gamma-} .
$$

Implementation of this twinning model to the plate impact tests gives results corresponding to the experimental observations, as it is shown in [15].

\section{Numerical implementation}

Equations of the twinning model, the dislocation plasticity model $[4,5]$ and the continuum mechanics equations with supplementary relations are numerically solved within 2D cylindrical version of the finite-deference computer code CRS, which $1 \mathrm{D}$ realization is briefly 


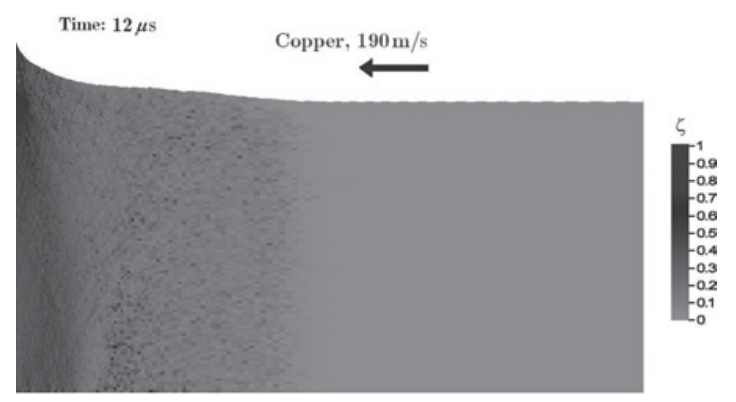

Figure 1. Shape of copper rod in the process of compaction with initial velocity of $190 \mathrm{~m} / \mathrm{s}$ (12 $\mu$ s from onset of impact) and spatial distribution of the volume fraction of twins.

described in [16]. Method of division on physical processes is used: each time step the continuum mechanics Eqs. (1)-(3) are solved at first and the macroscopic deformation (5) is defined, then the evolution of dislocations $[4,5]$ is calculated, then the evolution of twins (6)-(13), and, finally, the new stress field is determined with the use of the wide range equation of state [10] for pressure and Hook law (4) for deviators. The continuum mechanics equations are solved with using the finite-difference numerical method [17]. Equations of kinetics of dislocations and twins are solved predominantly by explicit Euler method.

\section{Results of calculations}

Modelling of the Taylor anvil on rod compaction of copper samples at various impact velocities (up to $500 \mathrm{~m} / \mathrm{s}$ ) was performed. Calculations shows, that the dislocation plasticity is predominant mechanism at low impact velocities $(<100 \mathrm{~m} / \mathrm{s})$, while twinning begins substantially influence at higher velocities.

Figure 1 presents an example of calculation results: the spatial distribution of the total volume fraction of twins at a moderate compaction velocity. Shock wave already passes through the sample. The twinning predominates in the part of the rod, which adjoins to the anvil; dislocation plasticity here is suppressed due to the hardening at the expense of twins. Dislocation plasticity is active in that part of the rod, in which the volume fraction of twins is low. Superposition of twinning and dislocation plasticity results in a complex form of the cross-sections of the deformed samples.

\section{Conclusions}

A kinetics-based model of the mechanical winning in metals at the high rate deformation is proposed. Growth of twins is considered as a collective slipping of the twinning dislocations; the finite rate of nucleation of twins is taken into account. The model of twinning is a complement to the dislocation plasticity model $[4,5]$ and requires only one additional parameter, which is the sacking fault energy.
The twinning model is applied together with the dislocation plasticity model to description of the plastic deformation in the dynamic Taylor anvil on rod tests. The obtained results have a qualitative correspondence with the experimental data about the form of the compacted samples. Calculations let us to reveal the contributions of the dislocation plasticity and twinning in different stages of the deformation process. Influence of twinning increases with the increase of the impact velocity. On the one hand, the twinning suppresses the dislocation plasticity due to the intense hardening of material in this area. On the other hand, the twinning provides an additional plastic deformation and extends the area of large deformations.

This study was supported by grants of the President of the Russian Federation (MD-286.2014.1) and the Ministry of Education and Science of the Russian Federation (State Task No. 3.1334.2014/K, Chelyabinsk State University).

\section{References}

[1] J.P. Hirth, J. Lothe, Theory of dislocations (Wiley \& Sons, New York, 1982)

[2] M.A. Meyers, K.K. Chawla, Mechanical behavior of materials (Cambridge University Press, New York, 2009)

[3] M.A. Meyers, D.J. Benson, O. Vohringer, B.K. Kad, Q. Xue, H.-H. Fu, Mater. Sci. Eng. A 322, 194 (2002)

[4] V.S. Krasnikov, A.E. Mayer, A.P. Yalovets, Int. J. Plast. 27, 1294 (2011)

[5] A.E. Mayer, K.V. Khishchenko, P.R. Levashov, P.N. Mayer, J. Appl. Phys. 113, 193508 (2013)

[6] J.W. Christian, S. Mahajan, Prog. Mat. Sci. 39, 1 (1995)

[7] E.N. Borodin, S.A. Atroshenko, A.E. Mayer, Tech. Phys. 59, 1163 (2014)

[8] G. Taylor, Proc. Royal Soc. London A, 194 (1948)

[9] M.L. Wilkins, M.W. Guinan, J. Appl. Phys. 44, 1200 (1973)

[10] V.E. Fortov, K.V. Khishchenko, P.R. Levashov, I.V. Lomonosov, Nucl. Instrum. Meth. Phys. Res. A 415, 604 (1998)

[11] L.D. Landau, E.M. Lifshitz, Course of Theoretical Physics Vol. VII Theory of Elasticity (Pergamon, New York, 1986)

[12] J.D. Eshelby, Collected works of J.D. Eshelby (2006)

[13] C. Kittel, Introduction to Solid State Physics (Wiley, New York, 2004)

[14] O.A. Plekhov, O.B. Naimark, N. Saintier, T. PalinLuc, Tech. Phys. 54, 1141 (2009)

[15] E.N. Borodin, A.E. Mayer, IOP Conf. Series: Mater. Sci. Eng. 63, 012034 (2014)

[16] A.E. Mayer, E.N. Borodin, V.S. Krasnikov, P.N. Mayer, J. Phys.: Conf. Ser. 552, 012002 (2014)

[17] A.P. Yalovets, J. Appl. Mech. Tech. Phys. 38, 137 (1997) 